\title{
Activités
}

12-1| Avril 2015

Varia

\section{Analyse de contextes d'activité domestique pour la conception de systèmes diffus énergétiquement efficients}

Analysis of domestic activity contexts for the design of ubiquitous energy-

efficient systems

Julien Guibourdenche, Jacqueline Vacherand-Revel, Myriam Fréjus et Yvon Haradji

\section{OpenEdition}

Journals

Édition électronique

URL : http://journals.openedition.org/activites/994

DOI : 10.4000/activites.994

ISSN : $1765-2723$

Éditeur

ARPACT - Association Recherches et Pratiques sur les ACTivités

\section{Référence électronique}

Julien Guibourdenche, Jacqueline Vacherand-Revel, Myriam Fréjus et Yvon Haradji, « Analyse de contextes d'activité domestique pour la conception de systèmes diffus énergétiquement efficients », Activités [En ligne], 12-1 | Avril 2015, mis en ligne le 15 avril 2015, consulté le 30 avril 2019. URL : http:// journals.openedition.org/activites/994 ; DOI : 10.4000/activites.994

\section{(c) (i) (9)}

Activités est mis à disposition selon les termes de la licence Creative Commons Attribution - Pas d'Utilisation Commerciale - Pas de Modification 4.0 International. 


\title{
Analyse de contextes d'activité domestique pour la conception de systèmes diffus énergétiquement efficients
}

\author{
Julien Guibourdenche
}

Université de Technologie de Troyes - ICD/Tech-CICO (UMR 6281 CNRS)

12, rue Marie Curie - BP 2060 - 10010 Troyes Cedex

julien.guibourdenche@utt.fr

\section{Jacqueline Vacherand-Revel}

\author{
École Centrale de Lyon/Laboratoire I.C.A.R (UMR 5191 CNRS - Lyon 2 - E.N.S Lyon - IFÉ) \\ 15, parvis René Descartes - BP 7000 - 69342 Lyon Cedex 07 \\ jacqueline.vacherand-revel@ec-lyon.fr
}

\section{Myriam Fréjus}

EDF R\&D - 1, avenue du Général de Gaulle - 92141 Clamart Cedex myriam.fréjus@edf.fr

\section{Yvon Haradji}

EDF R\&D - 1, avenue du Général de Gaulle - 92141 Clamart Cedex yvon.haradji@edf.fr

\begin{abstract}
Analysis of domestic activity contexts for the design of ubiquitous energy-efficient systems. From the standpoint of our situated approach to activity in cognitive ergonomics, this article discusses the design of ubiquitous energy-efficient computing systems in domestic settings. Drawing on doctoral research, our thesis is that such an ergonomic design requires an empirical understanding of three types of activity and context: the situated meaning of the action from the actor's point of view, the collective articulations of these meanings and the dynamics of physical arrangements. After briefly describing the design perspective and the theoretical and methodological framework developed, we argue in favor of this thesis in two stages. First, we show how our empirical results on the three dimensions feed design models (e.g. general principles, types of human-machine interaction to focus on, the type of contextual cues for the system...). We then show that our methodological advances and results allow us to explicitly formalize our design choices. The discussion opens up perspectives for further phenomenological "first person" approaches to human activity, and further characterizations of physical spaces in relation to possibility and impossibility of action in everyday settings.
\end{abstract}

\section{KEYWORDS}

Ubiquitous computing, Energy management, Context, Domestic activity, Methodology 


\section{Introduction}

L'efficience énergétique est aujourd'hui un enjeu incontournable du développement durable et concerne de plus en plus l'ergonomie. Notre recherche part du postulat selon lequel les systèmes informatiques diffus sont intéressants, parmi d'autres pistes possibles, pour réduire la consommation énergétique des bâtiments et des appareils (Harle \& Hopper, 2008; Meyers, Williams, \& Matthews, 2010). Un système informatique diffus se caractérise ici par deux traits : l'adaptation dynamique en temps réel à nos environnements et actions (e.g., Banâtre, Ciaran, Couderc, \& Weis, 2007) et l'élargissement de la palette d'interaction humain-machine (e.g., Cerisara \& Haradji, 2007 ; Coutaz, Crowley, Dobson, \& Garlan, 2005). Il s'agit donc d'un réseau d'objets répartis en plusieurs lieux, communiquant et/ou s'actionnant, qui peut potentiellement offrir plus d'efficacité énergétique (baisse des consommations), notamment en agissant directement sur les objets durant nos activités quotidiennes (Meyers et al., 2010).

Cependant, en ergonomie, l'efficacité énergétique (baisse des consommations) et les systèmes diffus peuvent poser des difficultés de conception en regard d'une efficience pour l'activité humaine (e.g., baisse des consommations respectant le caractère vivable des situations, le sens de l'activité...). Par exemple, si vous sortez d'une pièce, le système peut déclencher la coupure d'un ou plusieurs appareils (e.g., une ampoule), sur la base d'un indice contextuel physique (e.g., votre présence) sans que vous ayez l'intention d'éteindre à l'instant « $\mathrm{t} »$. La coupure n'est alors qu'indirectement en lien à votre intention de l'instant, en tant que celle-ci est elle-même liée à votre corps ou un ensemble d'effets physiques (e.g., les mouvements physiques du corps, la température d'une zone spatiale de la maison...). Mais puisque vous pouvez sortir d'une pièce avec d'innombrables intentions, besoins et contraintes, et que d'autres personnes peuvent évoluer en même temps dans le même espace, l'adaptation (et donc l'efficience) du système à votre activité n'est pas assurée. Par exemple, cette ampoule coupée vous était-elle utile lorsqu'elle était allumée ? Était-il pertinent de la laisser allumée pour un proche?

Comme le soulignent Zouinar \& Fréjus (2010), pour répondre à ce type de question dans une optique de conception de situations facilitant réellement la vie quotidienne, des évolutions théoriques et méthodologiques sont nécessaires, notamment pour mieux documenter les contextes d'activité humaine. Cet article vise donc à décrire les évolutions apportées par notre recherche, en en spécifiant les particularités de la conception de situations d'informatique diffuse visant l'efficience énergétique dans les habitats. Le système technique ne préexistant pas à notre recherche, nous nous plaçons dans une ergonomie en amont de la conception (e.g., Theureau \& Jeffroy, 1994), cherchant à développer des modèles instrumentant et guidant la conception technique. Après l'exposition des spécificités du cadre de conception, des choix théoriques et méthodologiques retenus, nous mettrons l'accent sur les liens entre les modèles empiriques et les options de conception à considérer. Nous proposerons enfin une formalisation des choix opérés pour la conception et reviendrons dans la discussion sur les évolutions nécessaires à l'avenir sur les plans empiriques et technologiques.

\section{1.- Positionner l'activité domestique et la consommation énergétique}

Notre recherche s'inscrit dans la continuité des travaux menés en ergonomie cognitive des situations domestiques (e.g., Fréjus, 2007a ; Haradji \& Faveaux, 2006 ; Haué, 2004 ; Poizat, Fréjus \& Haradji, 2012). Dans ce cadre, il s'agit pour nous de contribuer à la conception de situations d'informatique diffuse à la fois efficaces énergétiquement (baisse de consommation) et respectant les possibilités d'action des habitants (ou les renouvelant). Cela nous fournit une approche minimale de l'efficience énergétique. Cette visée de conception 
implique tout d'abord de relier la consommation énergétique à l'activité domestique de façon précise, poursuivant en cela les réflexions engagées par Fréjus (2007a) par exemple. La thèse que nous défendons est que la conception de situations informatiques diffuses efficientes énergétiquement nécessite trois types de connaissance, portant (a) sur la signification de l'action pour l'acteur humain, (b) sur l'articulation collective et (c) sur l'agencement physique des situations. Nos questions de conception peuvent être énoncées simplement comme suit. Dans quel contexte (individuel, collectif et physique) un système doit-il faire baisser la consommation d'un appareil électrique si l'on souhaite respecter les possibilités d'action significative pour l'humain? Dans quel contexte ne doit-il pas le faire, ou faire autre chose, et quoi ? Sur ces bases, quel type d'interaction humain-machine privilégier et sur quels éléments tangibles la machine peut-elle s'appuyer pour cela ? Plusieurs arguments créditent l'intérêt de cette approche.

Premièrement, l'activité domestique, envisagée à partir du point de vue des habitants, n'est pas orientée vers l'efficacité énergétique (e.g., Baillie \& Benyon, 2008 ; Chetty, Tran, \& Grinter, 2008 ; Martini et al., 2002), ou sinon très irrégulièrement (e.g., Haué, 2003). Ces travaux décrivent les préoccupations quotidiennes des membres des foyers, comme le soin aux enfants, l'organisation du vivre-ensemble, la réalisation des affaires quotidiennes... Certes, il y a bien des formes de finalités et de significations de l'action domestique en lien avec la régulation (baisse ou augmentation) des consommations d'énergie. Mais les études qualitatives (e.g., Chetty et al., 2008 ; Fréjus, 2007b ; Grosjean, Lacoste, \& Levy, 2001 ; Haué, 2003) comme quantitatives (e.g., I.F.O.P, 2009) montrent que le confort et les économies financières sont bien souvent les finalités les plus significatives pour les habitants, et pas nécessairement la régulation des consommations elle-même ou l'efficacité énergétique. En conséquence, s'intéresser au rapport entre consommation énergétique et action domestique nécessite de distinguer la signification de cette dernière par rapport à la genèse d'effets physiques de consommation énergétique. Par exemple, un habitant peut vouloir baisser le chauffage pour avoir moins chaud. Mais cela ne signifie pas que l'effet physique de baisse locale de consommation soit lié à une finalité significative d'efficacité énergétique chez l'habitant. Dans ces situations (e.g., la hausse/baisse de la température pour obtenir plus de confort), la consommation énergétique émerge comme effet indirect de la finalité significative d'action pour l'humain. En revanche, il faut aussi considérer qu'à d'autres moments, plus rares pour tout un chacun, ou plus fréquents peut-être pour des populations particulièrement sensibles aux enjeux écologiques (e.g., Barr \& Gilg, 2006), des significations et finalités de l'action émergent bien en lien avec l'efficacité énergétique ou avec des préoccupations environnementales.

Il est donc nécessaire de disposer de connaissances plus précises sur la signification de l'action pour les habitants, ainsi que sur l'environnement physique via le processus de consommation énergétique compris comme effet physique de l'activité avec les objets. Les modèles instrumentant la conception doivent nécessairement en tenir compte (par exemple pour définir des fonctionnalités et principes d'interaction cohérents), et nos modèles empiriques d'activité aussi puisqu'ils en forment le fondement.

Deuxièmement, notre thèse repose sur le fait que l'activité domestique possède de multiples dimensions collectives (e.g., conflits, coordination, négociations...), notamment reliées à l'utilisation des appareils électriques et de l'espace physique (e.g., Baillie \& Benyon, 2008 ; La Valle-Torres, 2011). Plusieurs modèles de système diffus dédié à la gestion énergétique dans l'habitat soulignent l'importance de cette dimension (e.g., Cerisara \& Haradji, 2007 ; Salembier, Dugdale, Fréjus, \& Haradji, 2009). Troisièmement, cette thèse s'appuie initialement sur les approches écologiques de l'habitat (e.g., Gibson, 1979 ; Moles \& Rohmer, 1973) sur lesquelles nous reviendrons plus spécifiquement en section 2.2. Ces travaux montrent que les situations domestiques sont d'une grande richesse sur le plan physique (e.g., plusieurs pièces, murs, étages...). Or, dans le cadre d'une conception de systèmes physiquement répartis dans l'habitat, cette richesse a tout lieu d'être une contrainte 
pour la conception si nos modèles n'en proposent pas une vision claire, systématique et articulée finement aux deux premières dimensions.

En conséquence, les modèles instrumentant la conception informatique doivent permettre d'articuler des dimensions contextuelles de l'activité qui sont significatives du point de vue des habitants individuellement et collectivement, ainsi que des dimensions contextuelles de l'espace physique (e.g., consommations énergétiques, mouvements, architecture...). Bien évidemment, d'autres dimensions importantes dans l'activité domestique pourraient être évoquées (e.g., histoire, culture...). Cependant, par souci de concision et de clarté de la thèse, nous nous sommes focalisés sur ces trois dimensions, tout en laissant ouvertes les éventuelles poursuites de celle-ci. Comme nous le verrons en section 6 , cela a finalement permis de proposer des orientations de conception technique en matière de finalité, d'objectifs et de principes d'interaction du système avec les habitants dans les situations futures, ainsi que des bases de définition des contextes pour la mise en œuvre de ces principes.

\section{2.- L'activité individuelle/collective entre significations et limites physiques}

Afin d'étayer empiriquement notre visée de conception, nous nous sommes appuyés sur les apports du cadre théorique du «cours d'action» (Theureau, 2004a) et sur notre propre développement théorique du « cours d'agencement » (Guibourdenche, 2013).

\section{1.- Préoccupations, cours d'action des habitants et articulation collective}

Le programme de recherche empirique du «cours d'action» (Theureau, 2004a) permet d'aborder les contextes d'activité individuelle et collective des habitants à partir d'une signification de l'activité vécue en situation par l'acteur. Quatre hypothèses ontologiques et deux objets théoriques sont retenus dans notre cadre.

Les deux premières hypothèses concernent l'énaction (Maturana \& Varela, 1992) et la conscience pré-réflexive (Sartre, 1943). Elles réduisent notre approche de l'activité des habitants à leurs aspects autonome, incarné et cognitif, à un sens construit en situation dont l'acteur est capable de témoigner moyennant les conditions méthodologiques favorables. La conscience pré-réflexive est considérée par Theureau (2006) comme un flux continu d'attentes, d'interprétations, de préoccupations, de ressentis et de focalisations évoluant en relation avec la situation en cours du point de vue de l'acteur. Selon la troisième hypothèse, l'activité collective consiste en une concaténation des activités individuelles-sociales. Le terme de "concaténation » souligne le caractère autonome de l'activité individuelle-sociale (e.g., la signification) au sein du couplage entre plusieurs acteurs.

Ces trois hypothèses impliquent de caractériser les contextes d'activité à partir de la signification de l'action et de l'articulation collective des significations construites par les habitants. Elles sont complétées par celle de la multiplicité et de la complexité des effets et contraintes de l'activité dans les corps, situations et cultures des acteurs (Sève, Theureau, Saury, \& Haradji, 2012; Theureau, 2011). L'activité humaine génère constamment des contraintes et effets sur les corps, situations et cultures. Comme le souligne Bourbousson (2010), cette hypothèse «est largement ouverte dans la mesure où elle ne spécifie pas $a$ priori la nature des contraintes qui pèsent sur l'activité, et des effets produits par cette activité » (p. 41). Elle s'apprécie selon les perspectives de conception des situations étudiées (domestiques, sportives...) et du (ou des) domaine(s) d'application (formation/apprentissage, entraînement, informatique...). C'est donc en fonction de ceux-ci qu'il est nécessaire de « questionner l'ensemble de ces contraintes et effets et leurs agencements » (Theureau, 2011, p. 4). Dans notre cas, elle ouvre sur le développement d'un objet/concept théorique particulier qui sort du domaine d'intérêt premier du programme de recherche empirique 
« cours d'action » : le « cours d'agencement » (voir section 2.2 suivante).

Dans le cadre du « cours d'action », l'analyse de l'activité ne peut se faire qu'« indirectement, c'est-à-dire à travers des objets théoriques, qui isolent certains de ses aspects » (Theureau, 2004a, p. 48). L'objet théorique «cours d'action» réduit l'activité à celle «d'un (ou plusieurs) acteur(s) engagé(s) dans une situation, qui est significative pour ce(s) dernier(s), c'est-à-dire montrable, racontable, et commentable par lui (ou eux) à tout instant, moyennant des conditions favorables» (Ibid.). Dans notre observatoire, la mise en œuvre des objets théoriques «cours d'action » et «articulation collective des cours d'action » nous permet de caractériser une partie des contextes d'activité tels qu'ils sont activement vécus et articulés par les habitants. En pratique, nous avons choisi de travailler plus particulièrement sur les liens entre action et « préoccupation ». Les travaux portant sur l'activité domestique (e.g., La Valle-Torres, 2011 ; Poizat, Fréjus, \& Haradji, 2009 ; Salembier et al., 2009) en ayant souligné l'importance, nous avons voulu approfondir ces connaissances et mesurer leurs conséquences sur nos visées de conception.

Enfin, le « cours d'action » nous aurait offert des voies d'accès empirique à l'agencement des contraintes et effets, en tant qu'ils sont significatifs du point de vue des habitants (e.g. Adé, 2005). Cependant, notre visée de conception nous a poussés à proposer des modèles contextuels d'activité rendant compte du domaine physique (et non plus cognitif) à partir (a) d'un point de vue plus extrinsèque sur l'activité et ses effets physiques dans l'espace de l'habitat, et (b) d'une analyse en terme de possibilité/impossibilité d'action/cognition.

\section{2.- Agencements des situations domestiques et cours d'agencement}

La construction du cadre du « cours d'agencement» vise une meilleure compréhension des conditions contextuelles dans lesquelles l'action et la cognition sont possibles ou impossibles (ce que nous synthétisons par la formulation « (im)possible ») dans l'interaction quotidienne entre les habitants et leurs situations (e.g., avec les objets quotidiens et avec les autres habitants) (Guibourdenche, 2013). Cela nécessite de faire l'hypothèse théorique selon laquelle les unités et les effets générés par les corps sur les situations interagissent, se lient et se délient à chaque instant, d'une façon spécifique et spécifiable par un observateur, qui ouvre simultanément sur des possibilités et des impossibilités d'action, cognition ou perception, pour les unités ultérieurement. Cette hypothèse est construite dans le champ de l'action/cognition située (e.g., Grosjean, 2005 ; Kaptelinin \& Nardi, 2006 ; Suchman, 2007). La notion d'« agencement » fait référence à une structuration précise, à un ordre relationnel ou encore un arrangement entre les éléments plus ou moins variés d'un environnement. Le concept de «cours d'agencement» a quant à lui été défini comme «la formation en cours des possibilités/impossibilités de perception, cognition et action, naissant des relations d'arrangement physique entre les unités formant l'environnement » (Guibourdenche, 2013, p. 309). Il ne s'agit donc plus de comprendre une actualité significative dans l'activité des habitants comme avec le «cours d'action». Il s'agit de comprendre la possibilité et l'impossibilité de cette actualité telles qu'elles sont (a) agencées dans/par la structuration de la situation antérieure (que les habitants ont contribué à transformer en partie) et (b) descriptible par l'analyste selon les conditions méthodologiques de l'observatoire.

Proches de notre domaine de conception, Dugdale \& Salembier (2008) ont cherché à définir dans quelle mesure une zone spatiale est visible ou non d'un habitant, certaines des modalités d'accès des agents à une pièce ou un appareil, à quelle condition l'on peut définir si une personne se trouve dans la pièce... Leurs références à la possibilité comme à l'impossibilité (zone non-visible, non accessible...) soulignent selon nous l'intérêt d'une réflexion généralisée sur ce sujet. Pour cela nous nous sommes inspirés de différents apports et débats au sein de l'action/cognition située (e.g., Béguin \& Clot, 2004 ; Grosjean, 2005 ; Theureau, $2004 b$ ) et de ses pourtours. Par exemple, l'inspiration du «cours d'agencement» par l'éthnométhodologie (Garfinkel, 1967) implique de nous intéresser à la disponibilité 
(descriptibilité, intelligibilité...) mutuelle des habitants participant à une interaction. Néanmoins, si les participants d'une conversation rencontrent des barrières contre lesquelles ils luttent (e.g., l'indexicalité, la réflexivité...), celles-ci ne résument pas la richesse des barrières se posant sur le chemin d'un habitant. Les habitats sont des sortes d'« enceintes partielles » (Gibson, 1979) c'est-à-dire des espaces semi-ouverts fondés sur des barrières physiques qui contraignent les corps humains, les possibilités de perception ou d'utilisation des espaces. En même temps ces barrières sont aussi une condition sine qua non de l'action et de l'appropriation du monde par l'humain (Gibson, 1979 ; Moles \& Rohmer, 1973), de la possibilité d'un sentiment de «chez soi» par exemple, de la simplification des actions possibles (Kirsh, 1995). L'inspiration du « cours d'agencement» par ces notions, croisée à notre ancrage dans l'action située, ouvre donc sur une prise en considération assez large de l'espace physique de l'habitat. Notre intérêt se porte sur la transformation physique en tant qu'ouverture/fermeture des (im)possibilités de l'activité domestique en situation, et sur la façon dont les habitants transforment l'espace, s'en émancipent ou le subissent.

L'agencement de la plupart des habitats et les déplacements des habitants nous amènent aussi à devoir faire l'analyse de l'activité d'un collectif familial en partie "éclaté ». Ce type de collectif, "contrairement aux situations coopératives classiques, n'est pas physiquement réuni en un même lieu au même moment» (Lacoste, 2001, p. 326). Paradoxalement, pour prendre en compte l'évolution dynamique et continue du collectif entre regroupement et éclatement, il faut s'intéresser plus précisément à la richesse locale des modalités de l'interaction par lesquelles ces effets sont générés sur la situation. Par exemple, «si les participants n'ont pas la possibilité de se voir », phénomène en jeu dans notre recherche, « l'interaction peut être organisée en référence à la seule parole, sans orientation vers les corps des participants ou vers un environnement physique partagé » (Goodwin, 2007, p. 60) ${ }^{1}$. Dans ce cadre, deux habitants peuvent être considérés comme co-présents au plan de la parole et des sons, alors qu'ils sont visuellement absents ou inaccessibles. Dans un autre cadre, un fait est manifeste pour un acteur s'il est perceptible ou inférable par un agent à un instant donné (Salembier \& Zouinar, 2006). Il peut donc être plus ou moins manifeste selon une condition, «un ensemble d'éléments qui vont déterminer le niveau de manifesteté mutuelle » (Salembier \& Zouinar, 2006, p. 66) d'évènements (e.g., des actions, l'affichage d'une information dans l'environnement, une alarme...). Par exemple, la persistance de l'évènement (e.g., un son dans l'environnement versus une inscription) ou la disponibilité des personnes nous intéressent au premier chef avec le «cours d'agencement» puisque nous cherchons à mieux comprendre comment les relations entre les unités formant un environnement ouvrent sur des (im)possibilités dans l'interaction entre les habitants et leurs situations. En synthèse, avec le «cours d'agencement », l'incarnation physique de l'activité n'est pas envisagée seulement dans ce qu'elle ouvre de possibilités d'action. Les réagencements physiques impliquent aussi de l'impossible à chaque instant.

\section{3.- Un observatoire original : du recueil au traitement}

Ces positions théoriques sur la signification de l'action et le monde physique ont de fortes conséquences méthodologiques, présentées succinctement dans cette section. Nous nous sommes appuyés à la fois sur l'observatoire du « cours d'action » et des méthodes permettant d'élaborer une analyse du « cours d'agencement».

1 Notre traduction de : «Thus, if participants cannot see each other interaction might be organized with reference to talk alone, without orientation to either the participants' bodies or to a shared physical environment »(Goodwin, 2007, p. 60). 


\section{1.- Recueil}

Les résultats présentés dans les sections suivantes s'appuient sur les données recueillies par Poizat et al., (2009) au sein de notre projet. Cinq foyers français $(\mathrm{N}=5)$ ont été sélectionnés afin d'accéder à une richesse des contextes d'activité, sur la base de trois critères principaux : la composition des foyers, le rapport à la technologie et les paramètres relatifs à l'habitat. Ces foyers répondaient également de profils à forte consommation électrique (habitats à étages, surface importante, au moins 3 membres dans le foyer, présence d'enfants, équipement technologique conséquent...).

Pour chaque famille, le dispositif de recueil comportait un entretien semi-directif, 3 enregistrements audio-visuels multi-spatiaux d'une durée de 6 heures chacun (sur des jours différents en terme d'activité comme les jours de semaine et de weekend) et des entretiens d'auto-confrontation menés en référence au «cours d'action » (Theureau, 2004a). Si cela permet l'analyse des « cours d'action », ces données audio-visuelles sont aussi cruciales pour permettre l'analyse des "cours d'agencement» dans l'activité domestique, cette dernière ayant lieu entre plusieurs pièces et plusieurs étages. Le nombre de pièces filmées en multispatial varie de 4 à 7 selon les familles, soit un total de 540 heures d'enregistrement audiovisuel.

Afin d'informer les participants des objectifs de la recherche, le cadre leur a été présenté plusieurs fois : a) au moment du recrutement par téléphone; b) au moment du premier contact à leur domicile puis c) lors du travail de terrain à leur demande. Afin de ne pas influencer de façon trop conséquente l'activité des habitants à propos des consommations énergétiques, la recherche a été présentée comme se focalisant sur les usages des appareils électriques dans une optique de conception de nouveaux services. Les familles étaient rémunérées pour cette étude, mais n'étaient pas tenues d'accepter d'être filmées dans toutes les pièces de leur maison, ni à toutes les plages horaires proposées. Les familles pouvaient contrôler l'enregistrement en temps réel via un interrupteur, ou en demandant à effacer les données (Poizat et al., 2009).

\subsection{Transcriptions multi-portées et formalisations pour les analyses empiriques}

Les premiers mois de la recherche ont été dédiés à un développement d'outil méthodologique de transcription qui a ensuite été utilisé pour la formalisation des « cours d'agencement » et des scénarios : le format multi-portées. L'enjeu initial était d'être capable de représenter de façon ramassée et articulée (a) les comportements de chaque individu en interaction avec sa situation, (b) les pièces et étages des habitats, (c) les verbalisations d'auto-confrontation et (d) les états de fonctionnement des appareils. Le format de transcription multi-portées y répond directement (Guibourdenche, Vacherand-Revel, Grosjean, Fréjus, \& Haradji, 2011), dans le cadre d'une analyse d'activité. Ce travail a notamment permis de produire 59 minutes de transcriptions multi-portées, à multiplier par des moments comprenant de 3 à 5 habitants, de 3 à 6 objets, entre 5 à 6 pièces d'habitat. Dans un premier temps, la famille Berrot ${ }^{2}$ et les moments à transcrire ont été sélectionnés sur la base de discussions collectives et de critères tels que (a) la variation de la présence de tous les membres dans l'habitat (e.g., lors des retours vers 17h30), (b) la présence de basculement entre des moments d'activité collective et plus individuelle (e.g., lors des couchers d'enfants) et (c) la présence de consommations électriques importantes, inférées sur la base d'observation des vidéos dévoilant l'état de marche de appareils. Nous avons ensuite réalisé des observations complémentaires dans les familles Crolle, Pollan et Mayol, de façon à comparer les résultats.

2 Toutes nos références au terrain et aux participants sont anonymes. 
À partir de ces transcriptions et observations complémentaires réalisées sur les vidéos et auto-confrontations nous avons tout d'abord réalisé une reconstruction des « cours d'action » individuels-sociaux des habitants via la méthode des récits réduits. Cette méthode, dont l'usage s'est répandu (e.g., Bationo-Tillon, 2006; Poizat, 2006) depuis les propositions initiales d'Haradji (1993), permet une analyse globale des «cours d'action » en structures significatives de différents rangs : des unités significatives élémentaires (USE) aux histoires, constituées par regroupement thématique. Nous avons ainsi formalisé 6 récits réduits d'habitants dans les familles. Au total nous obtenons un découpage des récits réduits en USE $(\mathrm{N}=926)$, séquences $(\mathrm{N}=212)$, macro-séquences $(\mathrm{N}=66)$ et histoires $(\mathrm{N}=23)$. Ce découpage indique que la ou les préoccupations qui «émergent» dans le moment considéré sont «de l'ordre de » telle ou telle signification pour l'acteur. Les unités de haut rang, comme les histoires, sont déterminées sur la base des unités significatives élémentaires et sur l'observation de leurs enchaînements (voir figure 1 pour une représentation graphique des histoires). Ceci a permis de caractériser et comparer formellement les contextes préoccupationnels dans lesquels les habitants se trouvent de leur point de vue. Ensuite, nous avons synchronisé les " cours d'action » de chaque habitant pour caractériser les contextes d'articulations collectives des préoccupations.

Afin de formaliser les «cours d'agencement », les préoccupations ont ensuite été intégrées à la transcription des comportements des habitants en format multi-portées (voir figure 5, chaque habitant possède une ligne " préoccupation » et « comportement »). Nous avons ainsi formalisé quatre-vingt-cinq agencements $(\mathrm{N}=85)$, émanant de quatorze moments $(\mathrm{N}=14)$ sélectionnés dans les cinq familles. L'objectif de comprendre la variété la plus grande possible des contextes d'agencement a guidé nos choix de moments à analyser. Par conséquent, une fois l'analyse d'un « cours d'agencement » terminée, nous avons recherché des moments soit radicalement différents, soit intéressants par la finesse des différences et similarités qu'ils présentaient par rapport au premier. Les analyses ont successivement porté sur l'agencement des (im)possibilités (a) d'interaction entre les habitants (notamment visibilité et audibilité mutuelles), (b) d'utilisation des objets et appareils du quotidien par les habitants et (c) des conditions d'(in)audibilité et d'(in)visibilité entre appareils et habitants. Nous reviendrons plus précisément sur les deux derniers dans la suite de cet article.

\section{4.- Résultats empiriques et réflexions technologiques}

L'analyse des données nous a permis de caractériser six types de résultats concernant: la signification des préoccupations des habitants, les anticipations qui y sont liées, l'articulation collective, les agencements-types, les barrières perceptives et la rigidité contextuelle des objets. Dans cette section, nous chercherons d'une part à présenter ces résultats, mais aussi à évoquer leurs implications pour la conception. Ces dernières ne se confondent pas avec les choix de conception formalisés en section suivante, bien qu'elles les orientent. Elles nous permettent d'expliciter notre thèse sur le rapport entre modèles empiriques et modèles pour la conception.

\section{1.- Une oscillation entre de multiples significations, mais pas d'efficacité énergétique}

Premièrement, sur les différentes unités significatives analysées, aucune n'est significativement liée à l'efficacité énergétique ${ }^{3}$. Nous n'observons pas, par exemple, d'unité dénommée «consommer moins d'électricité » ou «être plus efficace énergétiquement sur

3 Plusieurs raisons peuvent expliquer cette absence : le choix de ces foyers, de ces moments, ou le type d'analyse développé. Nous discuterons en fin d'article de ce résultat étonnant mais intéressant pour la conception. 
l'utilisation des lumières ». Ceci laisse entendre, dans la continuité d'autres travaux (e.g., Chetty et al., 2008 ; Martini et al., 2002), qu'en situation les habitants ne passent pas leur temps à gérer intentionnellement leur consommation énergétique. La figure 1 ci-dessous résume l'émergence des différentes préoccupations significatives dans le récit réduit du «cours d'action» de Mme Berrot, à l'échelle des histoires. Ces préoccupations sont représentatives du reste de celles étudiées dans les foyers, en tant qu'elles ne visent pas l'efficacité énergétique. Lors de sa soirée Madame Berrot va, par exemple, plutôt chercher à coucher ses filles, s'occuper du linge, dîner... C'est cela dont il s'agit de façon significative pour les habitants dans les familles étudiées en situation d'action, lorsqu'ils allument ou éteignent leurs appareils par exemple. Ainsi, Mme Berrot couchant ses filles va bel et bien allumer des lumières et un téléviseur, mais ceci afin de préparer le coucher de ses filles. De même, lors de l'extinction des feux à la fin du coucher, les lumières seront prises dans un contexte d'activité où Mme Berrot prend soin de ses filles et les met à l'aise pour la nuit. Elle n'éteint pas brutalement les lumières et reviendra entrouvrir la porte de la plus jeune... Le confort et l'affection sont alors plus prégnants que l'efficacité énergétique.

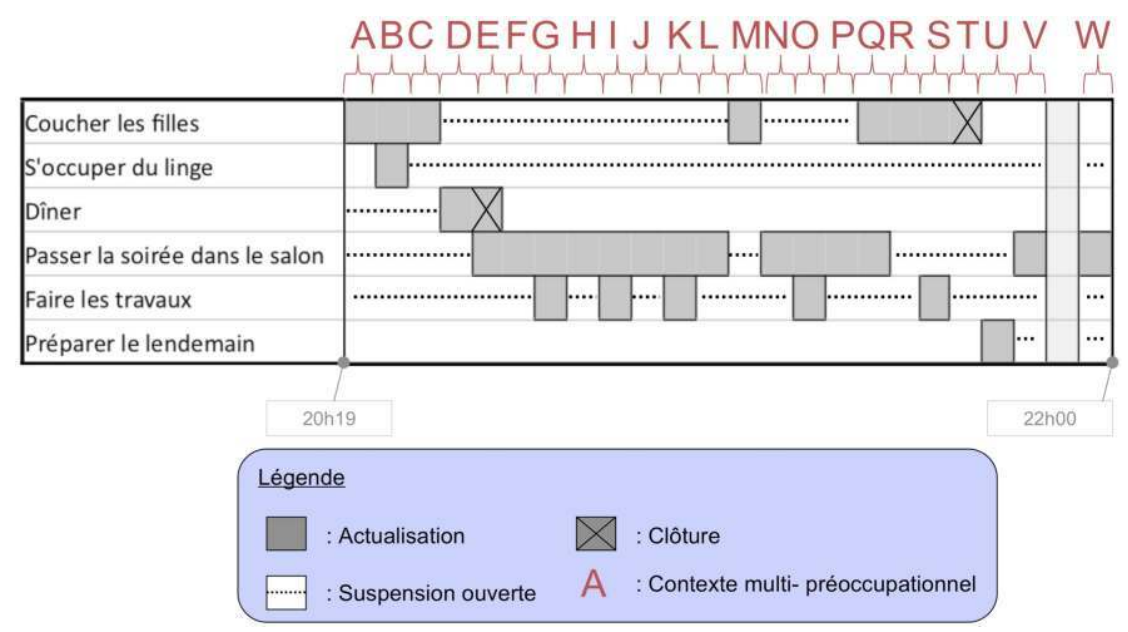

Figure 1 : Les contextes multi-préoccupationnels de Mme Berrot

Figure 1: Mrs Berrot's multi-concerns contexts

De plus, nous voyons sur cette figure que les préoccupations significatives des habitants émergent de façon discontinue dans le temps. Sur la figure 1, le contexte d'activité de Mme Berrot varie selon que la préoccupation est effectivement en cours de réalisation de son point de vue (nous dirons qu'elle est actualisée), suspendue et ouverte sur une continuation et une fin à venir (nous dirons qu'elle est suspendue-ouverte), ou encore effectuée pour partie ou en totalité (nous parlerons de clôture). Par exemple, la plupart des couchers d'enfants émergent de façon discontinue, mais conservent une cohérence d'ensemble à travers le temps du point de vue de l'acteur (e.g., préparer les chambres, préparer un biberon, coucher les enfants...). Cela s'effectue en lien à des évènements qui ouvrent sur des réorientations (e.g., sur la figure 1 , de $\mathrm{A}$ à $\mathrm{B}$, de $\mathrm{B}$ à $\mathrm{C}$, et ainsi de suite). Le basculement du contexte $\mathrm{L}$ à $\mathrm{M}$ est par exemple instigué à la suite d'un appel de Line, la fille de Mme Berrot, pour la préparation de son biberon. À d'autres moments, ce sont des évènements liés aux appareils qui vont ouvrir sur des basculements contextuels, comme des sonneries de téléphones et de fours, des fins d'émissions télévisées. Par exemple, le basculement ayant lieu à la fin du contexte $\mathrm{P}$ (voir figure 1 précédente) est lié à la fin d'une émission télévisée. Pour Monsieur Berrot à 16h35 le jeudi après-midi, un basculement marquant s'effectue à partir d'une sonnerie de téléphone de sa femme. M. Berrot interrompt alors la réalisation des travaux pour aider sa compagne à répondre au téléphone.

Que faire à partir de ce genre de résultats? Nous avons travaillé deux perspectives de conception complémentaires, impliquant des types d'interaction humain-machine différents (e.g., Dix, 2002). La première consiste à s'appuyer sur l'oscillation des significations de 
l'action et l'implication des appareils dans les basculements contextuels. On mobiliserait alors les supports techniques (e.g., téléphones, téléviseurs...) lors des basculements contextuels, pour proposer des possibilités d'action en faveur d'une gestion énergétique intégrée à l'existant. Ainsi, en proposant la possibilité de coupure d'un appareil ménager en plus des possibilités de répondre ou non à un appel téléphonique lors d'un appel entrant (e.g., Fréjus, Dominici, Weis, Poizat, Guibourdenche, \& Pietropaoli, 2013), on aiderait à faire des choix impactant l'efficacité énergétique en l'intégrant au sein d'un éventail élargi d'actions significatives possibles. La seconde perspective s'appuierait plutôt sur l'absence conséquente de significations liées à l'efficacité énergétique en action, qui mérite une attention très particulière. Cela mobiliserait plutôt un ensemble technique, à imaginer (voir Figure 8), qui aurait la charge d'assurer une plus grande efficacité énergétique sans faire appel constamment aux habitants. Quoi qu'il en soit, le développement de ces deux hypothèses doit prendre en compte les résultats empiriques suivants.

\section{2.- Des évènements anticipés à prendre en compte}

Les différentes préoccupations significatives sont en relation les unes avec les autres du point de vue des habitants. Certaines en empêchent ou gênent d'autres, leur font concurrence. Par exemple, faire des courses, repasser et suivre un feuilleton télévisé sont relativement difficiles à actualiser de front. D'autres, comme celles présentées dans la figure 2 suivante, sont en relation de nécessité. Ainsi, pour pouvoir passer une soirée dans le salon, les parents de la famille Berrot, ont à un moment besoin de coucher leurs deux filles.

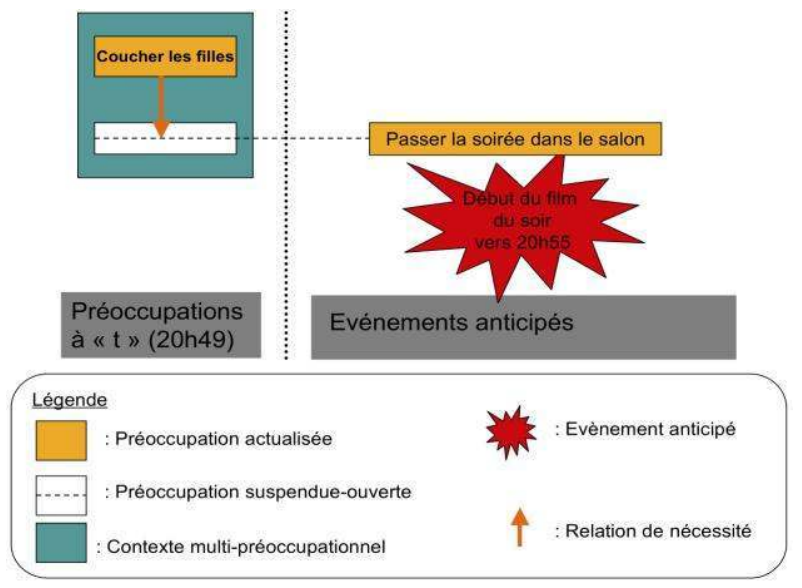

Figure 2 : Illustration d'anticipations lors du coucher des enfants

Figure 2: Illustration of anticipations while putting the children to bed

Du fait de ces relations, les événements anticipés par les habitants ne sont pas uniquement contextuels de la préoccupation qui les circonscrit, mais également d'une partie des autres préoccupations en relation (nécessité, concurrence ou agrément) avec la première. La figure 2 nous présente le cas du début imminent d'un film en soirée qui va être anticipé par les parents lors du coucher des enfants. On se trouve alors dans un contexte «multipréoccupationnel » habituel. Pour les parents de la famille Berrot le coucher du soir de Line et Jenny est habituellement structuré par un événement anticipé, à savoir le début du film du soir. Les parents vont coucher leurs filles vers 20h30-21h00, jusqu'à ce que le film commence pour eux. Le film s'insère lui-même dans une histoire où, généralement, les parents passent une soirée tranquille dans le salon (voir figure 1 précédente), suivant le film tout en lisant, jouant sur une console de jeux ou discutant en ligne selon les soirs (niveau de macro-séquence ou séquences non-représentables sur la figure 1).

Cet exemple fait partie d'une catégorie d'anticipation plus vaste et structurante pour la conception: les anticipations de retour à la préoccupation antécédente, impliquant très souvent un retour sur le lieu où se déroulait la préoccupation. L'exemple de la figure 5 , bien 
que représenté pour l'analyse des «cours d'agencement» en fait également partie. Mme Crolle va remonter coucher sa fille en suspendant ses préoccupations liées aux travaux d'aménagement et au suivi du film en bas dans le salon. Le corollaire régulièrement observé dans ces situations d'anticipation de retour est que les objets dont l'utilisation est anticipée sont laissés allumés (e.g., le téléviseur et les lumières du salon), en attendant de revenir.

Au plan de la conception, ces résultats impliquent que le système, d'une façon ou d'une autre, devra être capable d'anticiper sur des possibilités de retour. Il serait insuffisant de ne faire que couper les appareils (par exemple) que les habitants délaissent momentanément dans la suspension-ouverte des préoccupations significatives. Au contraire, il nous semble que le système devrait être capable de rallumer ce qu'il a lui-même coupé ou de permettre aux habitants de rallumer facilement ce qui a été coupé et au bon moment, par exemple. Sinon, il est assez certain que des erreurs seront commises, ouvrant sur des situations inconfortables (e.g., devoir rallumer tout ce qui a été coupé à chaque fois...), voire dangereuses (e.g., extinction de lumières dans des lieux comme les escaliers). De plus, cette anticipation, comme tout ce qui va déterminer la conception du système, doit se faire à l'échelle du collectif d'habitants et en prenant en compte les agencements physiques.

\section{3.- Similarité limitée des préoccupations dans le collectif}

L'analyse des articulations collectives des préoccupations individuelles des habitants nous montre formellement que les contextes d'articulation collective oscillent entre, d'un côté, un contexte de différence quasi-complète ${ }^{4}$, et de l'autre côté, un contexte de similarité quasicomplète entre tous les habitants (Guibourdenche, 2013). La différence quasi-complète se caractérise par des préoccupations différentes pour chaque membre, ce qui est rare. À l'inverse, la similarité quasi-complète se caractérise par des préoccupations d'ordre similaire, ce qui est également rare. L'articulation collective des préoccupations des habitants correspond le plus souvent à un contexte où 2 à 3 acteurs ont des préoccupations d'ordre similaire, tandis qu'un autre acteur actualise une préoccupation différente, ce que nous caractériserons comme « contextes de similarité limitée ».

\begin{tabular}{|c|c|c|c|}
\hline \multirow[b]{2}{*}{ Heure } & \multicolumn{3}{|c|}{$\begin{array}{l}\text { Préoccupations des acteurs* } \\
\text { (Séquences des récits réduits) }\end{array}$} \\
\hline & Pierre & Carine & Laura \\
\hline $\begin{array}{l}\text { De } \\
20 h 31 \mathrm{~m} 15 \mathrm{~s} \\
\text { à } \\
20 \mathrm{~h} 31 \mathrm{~m} 20 \mathrm{~s}\end{array}$ & $\begin{array}{l}\text { Discuter des } \\
\text { rideaux du lit } \\
\text { avec Carine }\end{array}$ & $\begin{array}{l}\text { Discuter des } \\
\text { rideaux sur le } \\
\text { magazine } \\
\text { avec Pierre }\end{array}$ & $\begin{array}{l}\text { Amener Papa } \\
\text { à regarder la } \\
\text { chanson du } \\
\text { dessin animé }\end{array}$ \\
\hline
\end{tabular}

N.B. Les couleurs vertes indiquent la similarité entre les préoccupations

Figure 3 : Articulation collective des préoccupations avec similarité limitée

Figure 3: Collective articulation of concerns with limited similarity

Par exemple, la figure 3 montre que, peu avant d'aller coucher leur jeune fille Laura, M. et Mme Crolle prospectent tous deux pour leurs travaux d'été s'orientant conjointement vers les rideaux et le magazine de travaux dans cette perspective. A contrario, Laura est simultanément en train de suivre un dessin animé près d'eux dans le salon et va chercher à amener son père à écouter la chanson du dessin animé.

Nous trouvons des expressions très variables de ce type de contexte selon les préoccupations

4 La particule « quasi » est utilisée pour tenir compte du fait que les contextes individuels étant multipréoccupationnels des similarités/différences peuvent persister pour d'autres préoccupations synchrones entre acteurs. 
en cours et le nombre d'habitants présents dans la maison. Il est par exemple possible d'observer une similarité entre trois acteurs, co-habitant avec deux acteurs dont les préoccupations respectives diffèrent de l'ensemble du collectif. Quoi qu'il en soit, nos résultats, comme d'autres dans la littérature (e.g., Baillie \& Benyon, 2008 ; Dimond, Fiesler, \& Bruckman, 2011 ; La Valle-Torres, 2011) démontrent une richesse à interroger finement, empiriquement. Dans notre cas, les contextes dévoilés oscillent sur des échelles temporelles de l'ordre de la seconde à la minute, entre un collectif «se réunissant à table pour manger plus ou moins ensemble (similarité quasi-complète)», et un collectif parfois en «coaction », parfois en « conflit », parfois loupant sa coordination...

Nous pouvons tirer deux conséquences de ces résultats pour la conception. Premièrement, si les contextes d'activité collective sont à similarité limitée, cela signifie que la conception doit créer un système capable de déterminer si un appareil est utilisé ou non (pour déclencher une fonctionnalité par exemple), mais collectivement et non pas individuellement. Un individu a beau ne pas utiliser un appareil, ces résultats signifient qu'il se peut qu'un autre l'utilise. Par conséquent, tout modèle de conception ne prenant en compte que l'activité individuelle ouvre sur une prise de risque non négligeable quant au respect des possibilités d'action des habitants et de l'utilisation du futur système (e.g., couper des lumières alors qu'elles étaient utilisées par d'autres). Deuxièmement, ces résultats impliquent qu'il est impossible, compte tenu de la complexité des contextes humains, de chercher à fournir un service « en tout lieu à tout instant $"$, chose pourtant visée dans certaines définitions techniques des systèmes diffus. Dans certains cas, il sera seulement nécessaire d'éviter d'interagir avec les habitants. Par exemple, s'il est possible qu'un appareil soit inutilisé par un habitant, ne pas gêner les autres habitants pourra consister à laisser faire, à ne pas intervenir si l'ambigüité sur le contexte des habitants est trop grande pour le système. À d'autres moments, il pourra s'agir de proposer des possibilités d'action aux habitants afin qu'ils puissent eux-mêmes couper certains appareils. Mais pour que le futur système soit capable de procéder de la sorte, nous avons besoin de comprendre les possibilités d'occupation/utilisation de l'espace physique en lien à ces préoccupations, anticipations, à l'activité collective des habitants. C'est pourquoi nous abordons à présent l'analyse des « cours d'agencement ».

\section{4.- Agencements-types identifiés : regroupé/éclaté, « en vue de »/effet secondaire}

Comme le résume la figure 4 suivante de façon très macroscopique, les collectifs d'habitants et leurs objets naviguent entre des agencements regroupés/éclatés entre les pièces de l'habitat. Cela signifie que les habitants, lorsqu'ils sont dans l'habitat, sont tantôt regroupés dans une même pièce, voire autour d'un espace commun (typiquement le repas familial lorsque tout le monde est réuni autour d'une table), tantôt seuls et isolés, chacun dans une pièce respective. En fait, on observe plus régulièrement des agencements intermédiaires impliquant par exemple deux personnes dans une même pièce et un ou deux autres dans des pièces ou étages séparés (voir Figure 6) également.

L'activité humaine et les situations engendrent ainsi des formes d'agencements variées au cours du temps, qui vont avoir des implications très fortes sur la possibilité et l'impossibilité d'interaction avec l'ensemble du collectif et des objets de la maison. Nos analyses permettent de distinguer deux façons dont peuvent être générés les effets sur l'environnement. Les agencements « en vue de » sont des effets fortement couplés aux préoccupations actualisées d'au moins un acteur, impliquant un contrôle fort de l'acteur sur sa situation. Par exemple, il peut s'agir d'aménager une zone «en vue du » repassage dans le salon, ce qui va impliquer un agencement local et évolutif du linge, de la table, éventuellement de l'éclairage, et aussi d'agréments comme la télévision ou une radio. 

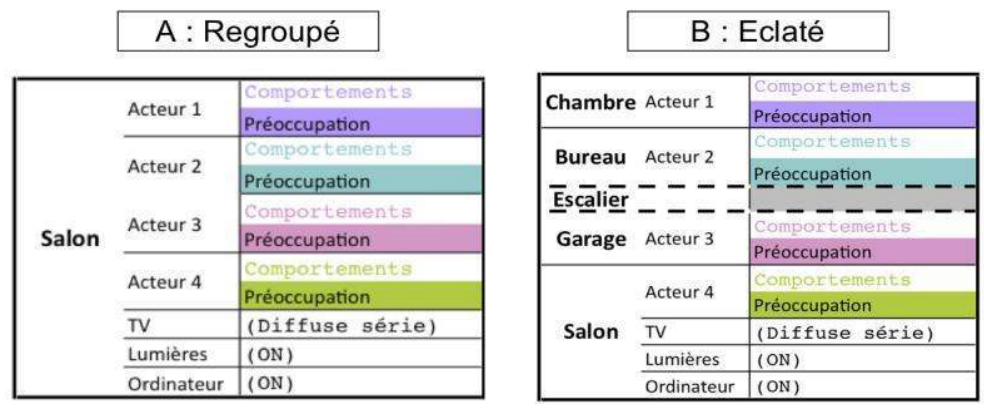

Figure 4 : Deux types d'agencements identifiés à l'échelle des pièces de la maison

Figure 4: Two typical "agencements" on a house room scale

Les agencements «par effets secondaires » sont, à l'inverse, uniquement tributaires d'une incidence fortuite du corps de l'acteur ou d'un objet, sur l'espace physique. Par exemple, les agencements de présence de la figure 4 sont principalement des effets secondaires, dans la mesure où la préoccupation de chaque habitant n'est jamais, selon nos résultats, « d'être dans telle pièce ». En revanche, le fait de se trouver dans telle ou telle pièce (e.g., dans la chambre au premier étage pour l'acteur 1 dans l'agencement B sur la figure 4 ci-dessus) a une conséquence pour l'agencement de possibilités dans l'activité collective et dans l'utilisation des appareils. L'acteur 1, selon l'agencement classique des habitats dans nos cultures, ne peut pas suivre l'acteur 4 et utiliser les appareils dans le salon. Ce type de résultat permet d'envisager quand et comment faire baisser la consommation de tel ou tel appareil sans gêner les habitants. Cependant, cette figure relève d'un point de vue trop générique pour cela et nous allons montrer en quoi les résultats détaillés importent pour la conception.

\section{5.- Les barrières perceptives et la continuité entre possibilité, ambiguïté et impossibilité}

Dans l'activité domestique, des agencements éclatés «humains-objets» limitent radicalement les possibilités d'interaction au plan sonore comme visuel pour le collectif, en l'occurrence ceux qui correspondent à un agencement regroupé du collectif autour d'un même espace et dans une même pièce.

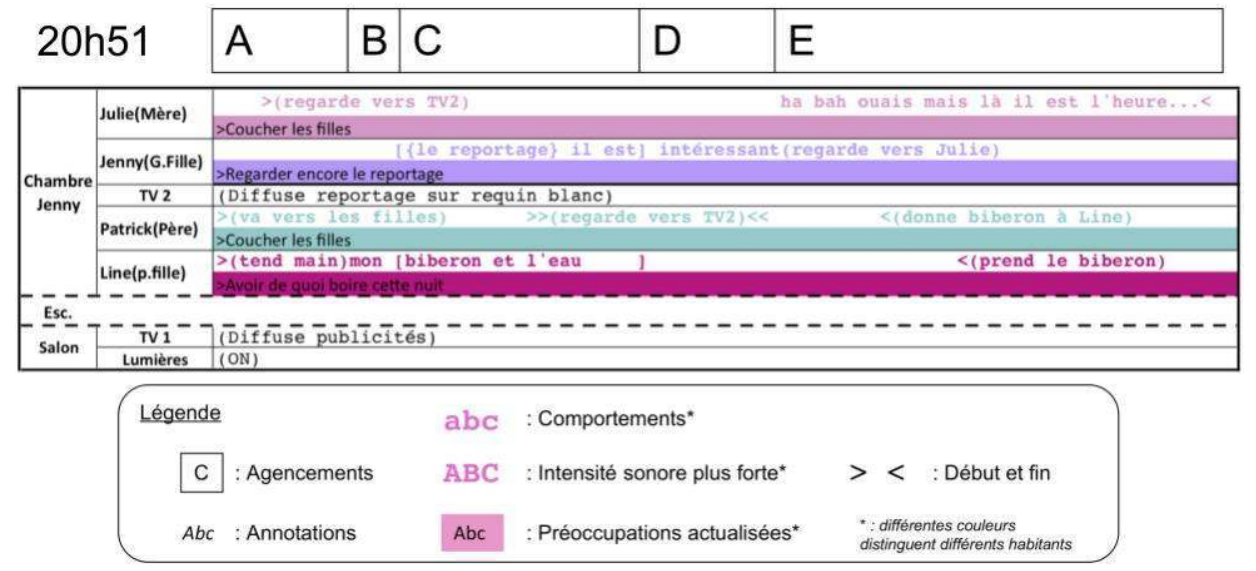

Figure 5 : Agencements « humains-objets » éclatés entre premier étage et rezde-chaussée

Figure 5: "Human-artifact" agencements scattered between first floor and ground floor

Par exemple, sur la figure 5 précédente, toute la famille Berrot est regroupée dans la chambre de Jenny lors du coucher des enfants. Cet agencement regroupe les membres de la 
famille et un ensemble d'objets dans la chambre au $1^{\text {er }}$ étage : la télévision $n^{\circ} 2$, diffusant un reportage sur le grand requin blanc, les lumières de la chambre, celles du couloir, des bouteilles d'eau, le biberon de Line... Mais il correspond aussi de façon centrale à un éclatement radical avec le téléviseur $n^{\circ} 1$ et les lumières du salon au rez-de-chaussée, qui ne peuvent pas être utilisés ni perçus dans ces agencements. En effet, des contextes d'agencements $\mathrm{A}$ à $\mathrm{E}$ (voir figure 5 précédente), l'éclairage du salon ne bénéficie pas à l'actualisation du coucher des filles, pas plus que le téléviseur $\mathrm{n}^{\circ} 1$. Le regroupement au premier étage sature l'espace sonore de telle sorte qu'une barrière perceptive acoustique émerge pour l'interaction avec ce même téléviseur $\mathrm{n}^{\circ} 1$. Le téléviseur de la chambre fonctionne, Line va s'adresser à son père pour prendre son biberon et sa bouteille d'eau... Puis très vite, en $\mathrm{C}$, Jenny s'adresse à sa mère pour tenter de regarder encore le reportage sur la télévision de la chambre. Les différents agencements sonores de la chambre ferment les possibilités de suivre à distance la télévision du salon. L'agencement des étages et des murs implique que l'écran du téléviseur et les lumières du salon sont «non-utilisés » ici, même si nous savons par ailleurs que les parents Berrot anticipent sur un retour dans le salon pour le début du film du soir (voir figure 2 précédente).

La caractérisation physique des (im)possibilités agencements permet plusieurs choses. Tout d'abord cela permet de guider la définition de dimensions sous-tendant ce que Cerisara et Haradji (2007) nomment les «contextes utile-machine ${ }^{5}$. Par exemple, sur la figure 5 précédente, le regroupement de tous en haut dans la chambre n'est pas propice à des possibilités de suivi à distance de l'écran de télévision en bas dans le salon, du fait de l'éclatement qu'il implique par effet secondaire. Cet éclatement est un contexte utile à la définition des impossibilités d'utilisation de l'écran du téléviseur $n^{\circ} 1$ en bas, par exemple. Nous entrevoyons alors la nécessité de baser le système sur la reconnaissance de présence du nombre d'habitants dans telle pièce, mais aussi des barrières visuelles ou des niveaux sonores locaux dans chaque pièce par exemple.

Ce type de résultats nous a aussi permis de définir des espaces abstraits de possibilité, ambiguité et impossibilité d'utilisation des appareils sur lesquels se base le système diffus dans nos scénarios (voir section 5). Cependant ces définitions nécessitent de comparer empiriquement un résultat comme celui de la figure 5 , simple, car radical, à d'autres qui le mettent en défaut par l'ambiguïté qu'ils dévoilent. Il existe notamment des agencements entre habitants et appareils qui apparaissent comme éclatés entre les pièces d'un même étage (donc ouvrant sur des impossibilités a priori), mais qui sont en fait regroupés au sein de l'espace d'interaction entre habitant et appareils. Par exemple, contrairement à des représentations bien ancrées dans notre culture, suivre un programme télévisé ne signifie pas nécessairement être devant son poste dans le canapé ni même dans la même pièce. Nous avons observé des contextes dans lesquels un habitant suit une série télévisée diffusée sur sa télévision dans le salon, alors que l'habitant se trouve dans la cuisine. Par conséquent, la fonctionnalité éventuelle de coupure d'un écran ne peut pas être mise en œuvre uniquement lorsque plus personne ne se trouve dans la pièce où est le téléviseur... La figure 6 de la section suivante montre qu'on peut aussi se trouver dans la même pièce qu'un téléviseur et ne pas le suivre. Ce sont ces types de comparaisons, démontrant en fait une continuité en transformation des possibilités, ambiguïtés et impossibilités d'utilisation des appareils, qui permettent d'affiner un modèle contextuel et dynamique des (im)possibilités d'utilisation de l'espace dans les scénarios de conception (voir figure 8).

5 Le contexte utile machine est un « contexte reconnaissable par une machine et utile pour adapter son action auprès de l'utilisateur » (Cerisara \& Haradji, 2007, p. 147). 


\section{6.- Rigidité contextuelle des objets quotidiens et gaspillage énergétique « situé »}

On peut enfin s'interroger sur la conception des situations domestiques en regard des possibilités de contrôle des consommations énergétiques. À partir de l'agencement $\mathrm{C}$ de la figure 6 suivante, Mme Crolle passera en tout 8 minutes en haut, « laissant » les lumières, la télévision, et l'ordinateur du salon en marche «alors qu'elle ne s'en sert pas ». Nous plaçons ces termes entre guillemets, car cette formulation linguistique fait porter la responsabilité de ces agencements à Mme Crolle.

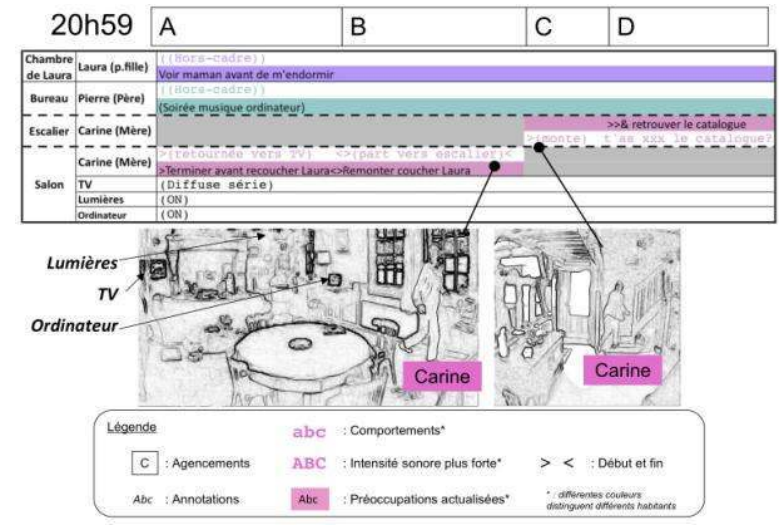

Figure 6 : Les objets restent allumés alors que plus personne ne les utilise

Figure 6: Appliances stay ON when nobody is using them

Or, pour éteindre, Madame Crolle (Carine sur la figure 6) aurait potentiellement à effectuer un déplacement vers chaque lieu de contrôle de ces objets, se trouvant éparpillés dans le salon et dans le couloir. Cela impliquerait aussi de tout rallumer à son retour. Cela serait un comportement tout à fait rationnel en regard de l'objectif d'efficacité énergétique, mais pas en regard de son activité significativement tournée vers le fait d'aller recoucher sa fille. Les objets et appareils «restent allumés » par " effet secondaire », mais cet effet secondaire ne peut pas être imputé uniquement au collectif des habitants selon nous. Dans ces agencements, le fonctionnement des objets est «contextuellement rigide». Les objets observés ne s'adaptent jamais aux déplacements des habitants, aux jeux de présence/absence ou d'agencement des (im)possibilités d'utilisation. Ainsi, aucun appareil ne s'éteint à partir de D sur la figure 6 ci-dessus, alors que l'utilisation en est impossible. Dans les familles Berrot, Crolle et Pollan, nous avons identifié 25 agencements avec barrières perceptives et appareils fonctionnant, sur une plage d'observation totale de $14 \mathrm{~h} 30$, impliquant en tout 33 objets du quotidien. Leurs durées varient de moins d'une minute à plusieurs heures. Par exemple, l'agencement éclaté débutant en $\mathrm{C}$ sur la figure 5 précédente durera 6 minutes. D'autres sont beaucoup plus longs. Ainsi, la télévision des Pollan sera mise en veille vendredi soir jusqu'au lendemain matin. Le samedi matin, après que Monsieur Pollan l'a rallumée avec la télécommande, elle fonctionnera encore dans un agencement radicalement éclaté durant 28 minutes de $9 \mathrm{~h} 43$ à $10 \mathrm{~h} 11$, lorsque la famille se prépare au premier étage. Nos analyses peuvent donc questionner de façon critique ce "gaspillage énergétique situé » entre les habitants et les concepteurs des objets ou des habitats. Les habitants ne peuvent pas tout à cet égard. La conception des appareils et des habitats est aussi largement en cause.

L'analyse des « cours d'agencement » nous permet donc de prendre du recul par rapport aux (im)possibilités d'action des habitants telles qu'elles sont agencées par l'espace physique. L'ensemble des résultats empiriques de cette section nous a permis d'établir des bases empiriques de réflexions portant sur les principes généraux de la conception. Sans prétendre offrir un guide pratique en section suivante, nous proposons des formalisations précises, permettant de discuter de nos choix sur des bases explicites et de continuer l'évolution théorique et méthodologique évoquée au début de cet article. 


\section{5.- Éléments formels de situations énergétiquement efficientes}

À partir de ces résultats, nous avons défini plusieurs principes de conception et d'interaction d'un système diffus dédié à l'efficience énergétique. Dans cette section, nous nous limiterons à spécifier globalement l'organisation du système et à présenter un scénario d'interaction future parmi trois conçus à l'aide du format « multi-portées ».

\section{1.- Organisation et principe d'aide à l'action sur les objets cibles}

La finalité d'efficience énergétique est réalisée par l'atteinte de quatre «objectifs 》 (voir figure 7) qui vont s'articuler ou prévaloir dans les situations futures selon les contextes détectés par le système: la possibilité d'action, le confort, la sécurité, et l'efficacité énergétique (baisse de consommation). Ces objectifs ne se valent pas dans notre conception. Le système devra faire progresser les possibilités d'action et la baisse des consommations énergétiques, et seulement ne pas nuire aux deux autres ${ }^{6}$.

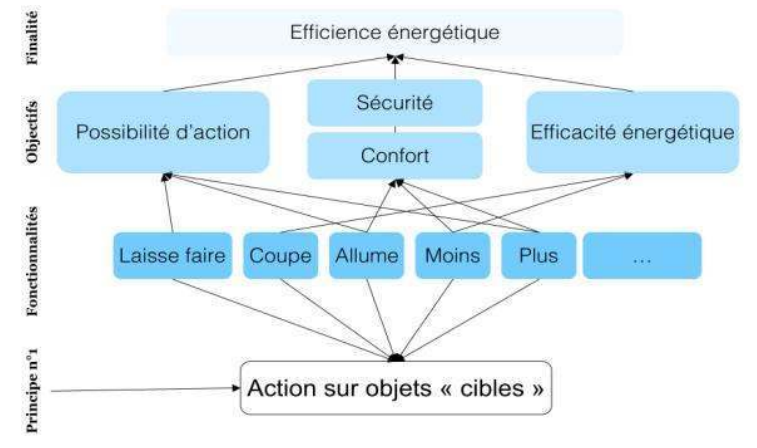

Figure 7 : Organisation générale du système en lien au principe $\mathrm{n}^{\circ} 1$

Figure 7: Overall system organization in relation to our first principle

Notre principe est de concevoir pour l'action, cela en visant des « objets cibles » et non des comportements humains. C'est pourquoi nous voyons sur la figure 7 que toutes les fonctionnalités ${ }^{7}$ sont tournées vers l'action sur des « objets cibles ». L'objectif de « possibilité d'action» vise alors les actions sur ces objets «cibles» (e.g., les ampoules, écrans, enceintes...) de façon répartie entre système technique et habitants. Premièrement, le système ne devra jamais entraver les possibilités d'actions des habitants, même si cela implique localement des effets de consommation d'énergie. Par exemple, dans les contextes où le système agit sans faire appel aux habitants, n'importe quel habitant doit pouvoir allumer ou éteindre ses lumières comme il l'aurait fait sans l'existence du système, ou ne pas subir de coupures inconsidérées des lumières. Deuxièmement, le système pourra proposer explicitement des possibilités d'action aux habitants sur les «objets cibles», renouvelant ainsi les possibles pour la gestion efficiente de l'énergie.

Opérationnellement, cela se traduit par la mise en œuvre contextuelle des fonctionnalités du système qui, du fait que l'on ne vise pas seulement l'efficacité, mais aussi trois autres objectifs, ne peuvent pas uniquement consister à couper ou réguler à moins de consommation (e.g., laisser faire, plus...). Selon les contextes pertinents pour le système, cette mise en ouvre variera. À travers la description d'un scénario d'interaction incidente ${ }^{8}$ (Dix, 2002), nous allons à présent envisager deux autres principes régissant la mise en œuvre

6 Il faut que l'efficacité énergétique ne se traduise pas par une mise en danger par exemple.

7 Ces fonctionnalités peuvent être actionnées à l'initiative du système ou des habitants grâce à un second ensemble de fonctionnalités (Guibourdenche, 2013).

8 Il existe une interaction incidente « lorsque des actions réalisées à d'autres fins, ou à partir de signaux nonconscients, sont interprétées dans le but d'influencer/améliorer/faciliter l'interaction future des acteurs ou la vie de tous les jours » (Dix, 2002, p. 2). 
contextuelle de ces fonctionnalités: l'aide locale/globale et le couplage aux contextes d'agencement.

\section{2.- Scénarisation des principes d'interaction}

Le scénario de la figure 8 nous permet d'envisager le fonctionnement d'un futur système à partir d'une évolution empiriquement réaliste des agencements d'un collectif entre plusieurs pièces et étages. Le système doit être efficace et respectueux des habitants quelles que soient les préoccupations de ces derniers. C'est pourquoi il est inutile de scénariser les préoccupations à ce stade.

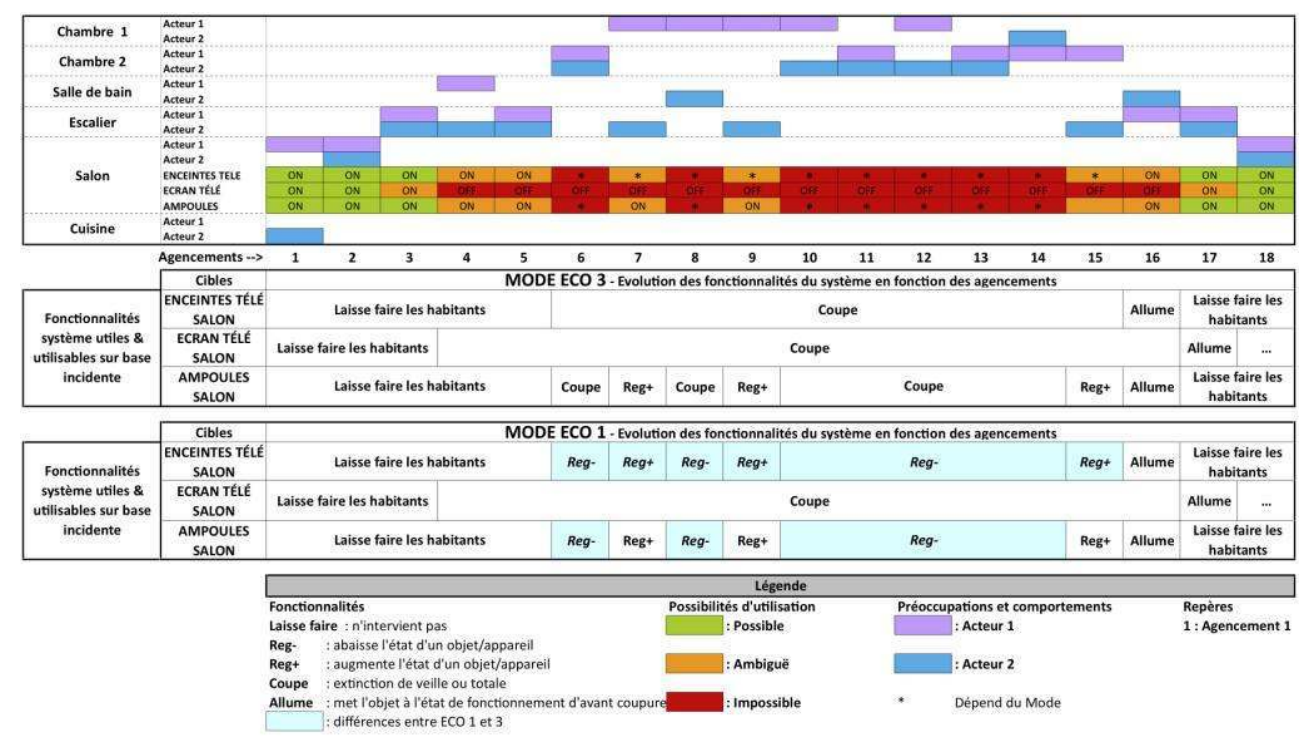

Figure 8 : Scénario d'interaction incidente

Figure 8: Incidental interaction scenario

Ce scénario regroupe en revanche des agencements très différents (éclatés, regroupés et intermédiaires), permettant d'envisager la dynamique d'évolution du futur système sur des bases riches, voire déjà compliquées pour la conception technique. Il est articulé autour de 18 agencements différents, numérotés de 1 à 18 . Entre ces agencements évoluent (a) la présence de deux habitants dans l'habitat, et (b) les possibilités, ambiguité et impossibilité d'utilisation de trois cibles : écran de télévision, enceintes et ampoules du salon.

Sur cette base, nous concevons premièrement des aides locale et globale sur les plans temporel et physique. Sur le plan temporel, l'aide locale est réalisée lorsqu'une fonctionnalité efficace et respectant les possibilités d'action en contexte est activée par le système ou l'habitant dans l'instant «t $\mathrm{t}$ (e.g., dans l'agencement 9 l'écran est « coupé » par le système au rez-de-chaussée), tout contexte d'agencement pertinent par ailleurs. Ici un acteur monte dans l'escalier, l'autre est dans la chambre 1 donc la fonctionnalité de coupure de l'écran est nongênante, car il est impossible d'utiliser l'écran. Une aide globale dans le temps, consiste par exemple à anticiper les retours possibles pour gérer les « objets cibles » au proche retour des habitants dans le rez-de-chaussée (e.g., agencement 16).

Sur le plan spatial physique, une aide locale est réalisée lorsque le système ou l'habitant actionne une fonctionnalité sur un seul objet cible (e.g., l'écran télé). Dans ce cadre, c'est bien une aide globale physique qu'il faut développer (e.g., avec les trois objets cibles du salon, pris pour exemple). Cette aide doit permettre de maximiser le nombre d'objets cibles sur lesquels actionner les fonctionnalités (notamment «coupure» et "Reg-»), tout en empêchant les barrières à l'action, les possibilités d'inconfort et d'insécurité pour les habitants. 
C'est aussi pourquoi nous regroupons les fonctionnalités en «modes » de fonctionnement (voir les deux cadres « Mode ECO » 3 et 1 sur la figure 8 précédente). Un mode regroupe un ensemble d'objets cibles actionnables et des fonctionnalités types à mettre en œuvre sur cet ensemble selon les contextes d'activité du point de vue du système. Cela va permettre d'agir sur plusieurs objets simultanément, de donner une logique d'ensemble à l'aide apportée aux habitants, tout en favorisant plus ou moins d'efficacité énergétique selon les choix des habitants. Par exemple, le mode ECO 3 va maximiser les opportunités d'efficacité énergétique tout en prenant en compte les contextes d'agencement pour atteindre les trois autres objectifs. C'est pourquoi il comporte plus de fonctionnalités de coupure qu'ECO 1 (voir Figure 8 précédente, les cases bleu clair différenciant les deux modes selon les fonctionnalités). Il ne peut pas comporter uniquement des fonctionnalités de coupure, notamment lorsque les personnes passent du ler étage aux escaliers. Le système va devoir augmenter la luminosité dans l'escalier (pour éviter des dangers potentiels de chutes, par exemple) en agissant sur les lumières si d'autres sources lumineuses ne sont pas effectives (e.g., « Reg+ » à l'agencement 7). C'est pourquoi l'aide doit être à la fois locale et globale.

Nous voyons donc comment nos deux premiers principes (action sur les « objets cibles » et aide locale/globale) peuvent s'incarner au sein d'un scénario d'interaction incidente. Nous ne présenterons pas le principe d'interaction incidente/intentionnelle ici et terminerons en indiquant sur quelle base la mise en œuvre « effective » des fonctionnalités peut être pensée. Ceci implique notre quatrième principe de conception spécifiant des bases physiques à la définition de «contexte-utile machine» (Cerisara \& Haradji, 2007): le couplage aux agencements physiques. Afin de définir quel objectif prioriser, de définir les objets cibles, le type de fonctionnalité à mettre en œuvre et le type de base d'interaction, le système devra être capable d'évaluer quelque chose de l'ordre d'une (im)possibilité d'utilisation des objets/espace par le collectif, sur une base de captation d'états physiques. C'est pourquoi, à partir de nos résultats, nous définissons formellement, pour chaque cible, des «espaces abstraits " d'utilisation et les bases physiques de leur définition: les espaces d'utilisation possibles (E.U.P), impossibles (E.U.I) ou ambiguë (E.U.A). Ce qui devra importer pour le système est qu'un habitant ou un groupe d'habitants puisse ou non se trouver dans de tels espaces : en train d'utiliser ou non tel objet ou un espace physique spécifique. Par exemple, dans la figure 8, les habitants sortent physiquement du salon de 1 à 8 , entrant simultanément et progressivement dans l'E.U.A puis l'E.U.I des cibles du rez-de-chaussée. Pour définir ces espaces, nos résultats sur les "cours d'agencement», nous avons vu que nos résultats nous permettent de spécifier un ensemble de contextes-utiles machines (Cerisara \& Haradji, 2007) en discussion avec la conception technique, comme par exemple le sens physique d'un déplacement, la présence de barrières perceptives visuelles ou acoustiques... Ces éléments sont formalisés ailleurs? ${ }^{9}$.

Nous avons donc montré en quoi les formalisations de situations futures pouvaient s'appuyer sur nos résultats empiriques. Des versions antérieures de ces modèles ont servi de base à des discussions lors d'étapes intermédiaires du projet, entre ingénierie informatique et ergonomie. Nous pouvons à présent conclure et proposer des axes de développement ultérieur.

\section{Conclusion et discussion}

La thèse sous-tendant cet article et notre recherche voulait que la conception de situations informatiques diffuses efficientes énergétiquement nécessite trois types de connaissance, portant (a) sur la signification de l'action pour l'acteur, (b) sur l'articulation collective et (c)

${ }^{9}$ Cf. Guibourdenche (2013) 
sur l'agencement physique des situations. Sa mise à l'épreuve dans nos recherches nous semble montrer à quel point les appels à une réflexion théorique et méthodologique sur la documentation des contextes d'activité humains (e.g., Zouinar \& Fréjus, 2010) doivent être entendus et poursuivis. Nous avons vu que notre objectif de conception nécessitait de développer un cadre théorique et méthodologique particulier, ancré dans les apports de l'action/cognition située mais cherchant aussi à se développer de façon spécifique (e.g., «cours d'agencement», format multi-portées...). Nous avons montré les implications technologiques de résultats empiriques portant sur les préoccupations, leurs articulations collectives et les agencements de l'activité quotidienne. Ces résultats permettent de réfléchir tout autant aux principes de conception à mettre en œuvre, au type d'interaction humain machine à privilégier en contexte, qu'au type de contexte-utile machine ou d'indice sur lequel s'appuyer concrètement. Ils permettent aussi de proposer des abstractions utiles à la conception comme les E.U.P, E.U.A et E.U.I. In fine, le travail de développement méthodologique du format multi-portées, initialement développé pour la transcription, nous aura permis de réinjecter les éléments empiriques et technologiques au sein de scénarios qui formalisent explicitement les choix de conception.

Nous voudrions ouvrir deux plans de discussion pour les futures recherches empiriques à visée technologique. Premièrement, bien qu'apportant déjà des éléments intéressants, nos recherches n'ont fait selon nous qu'effleurer le «domaine cognitif» (Maturana \& Varela, 1992) des habitants dans l'activité domestique, via la caractérisation des préoccupations et anticipations significatives pour les habitants. À l'avenir, il sera intéressant de travailler à enrichir la description en première personne de l'expérience des habitants (e.g., Cahour, Brassac, Vermersch, Pachoud, \& Salembier, 2007 ; Theureau, 2006). Nous pouvons pointer trois axes à cet égard. Concernant la cognition vécue en situation et l'efficacité énergétique, un travail plus long sera nécessaire afin de mieux positionner leurs relations. Par exemple, on pourra chercher à analyser plus finement les cours d'expérience des habitants afin de comprendre la conscience pré-réflexive (Theureau, 2006) des habitants de façon plus profonde (e.g., actualité potentielle, représentamens, interprétations...). On peut aussi se demander si à certains moments, l'efficacité énergétique ne serait pas une sorte de préoccupation sous-jacente des individus, comme «pré-réfléchie » (Vermersch, 2012), mais en mal de situations permettant leur réalisation. Ces points paraissent importants à traiter en lien avec nos postulats initiaux et demanderont des avancées méthodologiques et empiriques nouvelles. Le deuxième axe concerne le paysage émotionnel et affectif de l'activité des habitants. Nous n'en avons à ce jour qu'une idée très partielle, bien que nos analyses nous aient parfois menés près de certains sentiments et émotions (peurs, joies...). Des travaux sur les émotions et les affects des habitants approchés en "première personne » permettraient par exemple de préciser les valences ou les polarisations des préoccupations des habitants par rapport à notre travail, ou des sentiments associés à l'utilisation de l'espace. Dans la mesure où la confiance des habitants dans leur système et leur fournisseur d'énergie est aujourd'hui reconnue comme l'un des facteurs importants pour l'efficience énergétique (Rodden, Fischer, Pantidi, Bachour, \& Moran, 2013), ces progressions nous semblent nécessaires. Cela permettrait aussi de réfléchir différemment à des dimensions technologiques clés comme la sécurité ou le confort. Enfin, un troisième axe de progression est l'échelle de projection spatio-temporelle des activités, en jeu dans les anticipations, mais aussi certainement dans l'appropriation de l'espace physique. Nous avons travaillé à l'échelle de plusieurs heures avec un collectif à l'intérieur de la maison, ce qui représente déjà un apport. Mais il sera nécessaire d'envisager des temps et des espaces plus vastes à l'avenir. Nous pensons que nos travaux offrent des bases théoriques, empiriques et technologiques à ces futurs travaux, et qu'il sera intéressant de suivre l'évolution des perspectives de conception. Ceci nous amène au deuxième plan de discussion à propos du cours d'agencement, qui représente une forme de projection analytique particulière de l'activité collective et de l'espace physique. 
Le «cours d'agencement» est une construction conceptuelle ayant trouvé sa pertinence à travers nos questions de conception pour l'activité domestique et l'efficience énergétique. Mais l'efficience énergétique et les systèmes diffus concernent plus largement l'activité humaine future. Il sera donc intéressant de discuter plus largement les postulats du «cours d'agencement» concernant la transformation des «(im)possibilités» dans d'autres domaines, comme les organisations de travail (e.g., Lorino, 2009), l'interaction humainmachine (e.g., Ramduny-Ellis, Dix, Gill, \& Hare, 2009), ou encore la conduite automobile (e.g., Munduteguy \& Darses, 2007) par exemple. Le «cours d'agencement » offre, après les réflexions de Grosjean (2005) notamment, une manière d'aborder les "(im)possibilités » dans l'activité à partir des transformations et agencements de l'espace physique. Cela ne signifie pas que l'on nie la dimension culturelle, sociale et historique de l'activité. En revanche, nous mettons fortement l'accent sur la transformation de l'espace physique. Le « cours d'agencement», particulièrement à travers sa formalisation en portées multiples, nous paraît résonner avec certaines propositions de Suchman (2007) concernant l'agency (capacité d'action) et son traitement méthodologique. Suchman (2007) constate une extension problématique des cadres d'analyse souhaitant investiguer des réseaux composites entre humains et non-humains tout en conservant un ancrage fort à la dissymétrie, à la situation et à l'incarnation des relations humain-machine. Elle souligne alors qu'il est nécessaire d'effectuer un "zoom arrière », tout en portant " [...] attention aux questions de cadre, au travail de délimitation à travers lequel une entité donnée est délinéée en tant que telle » (Suchman, 2007, p. 283) ${ }^{10}$. C'est ce que nous avons cherché à faire avec l'observatoire $\mathrm{du}$ « cours d'agencement », en élargissant l'approche de l'activité collective sur le plan spatial physique, et ceci toujours à partir des situations locales et d'un travail de cadrage théorique et méthodologique reconnaissant le rôle actif du chercheur dans cette « délinéation ». À ce titre, la distinction entre approches intrinsèque et extrinsèque de l'activité (e.g., Theureau, 2006) nous paraît être importante dans ce "découpage » mêlant différents points de vue, sur les plans empirique et technologique.

L'échelle pertinente pour la conception des situations en jeu dans nos recherches sera donc de plus en plus «à situer localement au sein d'un cadre d'analyse élargi ». Par conséquent, il restera nécessaire d'étendre et enrichir les cadres de l'action/cognition située et ceux de l'ergonomie de langue française dont elle est proche sur bien des aspects (Theureau, 2004b). Avec cet article, nous espérons avoir apporté certains éléments structurants à cet égard. Dans la mesure où l'efficience énergétique est un défi pour notre siècle et pour l'ergonomie, il nous semble que de telles réflexions doivent être poursuivies de façon contradictoire et ouverte.

\section{REMERCIEMENTS}

Cette recherche doctorale a été réalisée en Convention Industrielle de Formation par la REcherche, de janvier 2009 à janvier 2012 (http://www.anrt.asso.fr). Elle a été effectuée en collaboration entre le laboratoire Interactions, Corpus, Apprentissages \& Représentations (UMR 5191 CNRS - Lyon 2 E.N.S Lyon - IFÉ) et Électricité de France - Recherche \& Développement. Nous tenons à remercier spécialement Michèle Grosjean, Professeure Émérite à l'Université Lyon 2, pour son travail au sein de cette collaboration, et la direction de la recherche doctorale qu'elle a assurée jusqu'à sa disparition en juin 2011. Nous sommes également reconnaissants envers le Professeur Yves Clot pour son soutien et ses remarques, ainsi qu'à Germain Poizat pour sa collaboration, en particulier pour le recueil de données.

\section{Bibliographie}

Adé, D. (2005). L'agentivité des objets ordinaires dans la leçon d'éducation physique et sportive: une

10 Notre traduction de : «It demands attention to the question of frames, of the boundary work through which a given entity is delineated as such. » (Suchman, 2007, p. 283) 
analyse à partir de l'étude de l'activité d'enseignants stagiaires. Thèse de doctorat en STAPS, Université de Rouen, France. https://apprendreeneps.files.wordpress.com/2013/07/thc3a8se-davidadc3a9-2005.pdf

Baillie, L., \& Benyon, D. (2008). Place and technology in the home. Computer Supported Cooperative Work, 17(2-3), 227-256.

Banâtre, M., Ciaran, B., Couderc, P., \& Weis, F. (2007). Informatique diffuse : des concepts à la réalité. Paris: Lavoisier.

Barr, S., \& Gilg, A. (2006). Sustainable lifestyles: Framing environmental action in and around the home. Geoforum, 37(6), 906-920.

Bationo-Tillon, A. (2006). Pratiques des activités narratives instrumentées. Une analyse diachronique et structuro-fonctionnelle en amont de la conception. Thèse de doctorat d'Ergonomie, Université Paris 8, Saint-Denis, France. https://tel.archives-ouvertes.fr/tel00096993/document

Béguin, P., \& Clot, Y. (2004). L'action située dans le développement de l'activité. @ctivités, 1(2), 3549.

Bourbousson, J. (2010). La coordination interpersonnelle en basketball. Ergonomie cognitive des situations sportives. Thèse de doctorat en STAPS, Université de Nantes, France. http://www.univnantes.fr/bourbousson-j/0/fiche__annuaireksup/

Cahour, B., Brassac, C., Vermersch, P., Pachoud, B., \& Salembier, P. (2007). Étude de l'expérience du sujet pour l'évaluation de nouvelles technologies : l'exemple d'une communication médiée. Revue d'anthropologie des connaissances, 1(1), 85-120. doi:10.3917/rac.001.0085

Cerisara, C., \& Haradji, Y. (2007). Nouvelles formes d'interaction homme-machine pour l'informatique diffuse. In Informatique diffuse (pp. 129-157). Paris: Observatoire Français des Techniques Avancées.

Chetty, M., Tran, D., \& Grinter, R. (2008). Getting to green: Understanding resource consumption in the home. In UbiComp'08, September 21-24 Seoul, Korea: ACM DL.

Coutaz, J., Crowley, J., Dobson, S., \& Garlan, D. (2005). Context is key. Communications of the ACM, 48(3), 49-53.

Dimond, J. P., Fiesler, C., \& Bruckman, A. S. (2011). Domestic violence and information communication technologies. Interacting with Computers, 23(5), 413-421.

Dix, A. (2002). Beyond intention: Pushing boundaries with incidental interaction. Presented at the Building Bridges: Interdisciplinary Context-Sensitive Computing, Glasgow. http://alandix.com/academic/papers/beyond-intention-2002/

Dourish, P. (2004). What we talk about when we talk about context. Personal Ubiquitous Computing, $8(1), 19-30$.

Dugdale, J., \& Salembier, P. (2008). Modélisation située des activités individuelles et collectives pour la conception. Clamart: EDF R\&D.

Fréjus, M. (2007a). Analyse ergonomique des pratiques domestiques pour la conception de situations de vie innovantes : Un exemple avec l'activité de cuisine. In Actes du 42ème congrès de la SELFErgonomie des produits et des services (pp. 91-100). Toulouse: Octarès. http://www.ergonomieself.org/media/media41277.pdf

Fréjus, M. (2007b). Les questions de société comme nouveau territoire pour l'ergonomie : apports aux problématiques environnementales et à la conception de services associés. In Actes du 42ème congrès de la SELF-Ergonomie des produits et des services (pp. 33-41). Toulouse: Octarès Editions. http://www.ergonomie-self.org/media/media41277.pdf

Fréjus, M., Dominici, M., Weis, F., Poizat, G., Guibourdenche, J., \& Pietropaoli, B. (2013). Changing interactions to reduce energy consumption: Specification of a context-aware system centered on the home occupants' concerns. In C. Stephanidis (Ed.), HCI International 2013 (pp. 638-642). Berlin: Springer. http://link.springer.com/chapter/10.1007/978-3-642-39476-8_128

Garfinkel, H. (1967). Recherches en ethnométhodologie (2007th ed.). Paris: Presses Universitaires de France.

Gibson, J. J. (1979). The ecological approach to visual perception (1986th ed.). New York, N.Y: Psychology Press. 
Goodwin, C. (2007). Participation, stance and affect in the organization of activities. Discourse \& Society, 18(1), 53-73. doi:10.1177/0957926507069457

Grosjean, M. (2005). L'awareness à l'épreuve des activités dans les centres de coordination. (a)ctivités, 2(1), 76-98.

Grosjean, M., Lacoste, M., \& Levy, E. (2001). Approche ethnographique et socio-pragmatique des nouveaux services interactifs (Rapport interne). Clamart, France: EDF R\&D.

Guibourdenche, J. (2013). Préoccupations et agencements dans les contextes d'activité domestique. Contribution à la conception de situations informatiques diffuses, appropriables et énergétiquement efficaces. Thèse de doctorat en Psychologie Sociale et du Travail, Université de Lyon, France. https://tel.archives-ouvertes.fr/tel-01068697

Guibourdenche, J., Vacherand-Revel, J., Grosjean, M., Fréjus, M., \& Haradji, Y. (2011). Using multiple scores for transcribing the distributed activities of a family. In Proceedings of the ACM 2011 conference on Computer supported cooperative work (pp. 637-640). New York, NY, USA: ACM. doi:10.1145/1958824.1958933

Haradji, Y. (1993). De l'analyse de l'aide humaine à la conception d'une aide informatique à l'utilisation de logiciel. Thèse de doctorat d'Ergonomie non publiée, Conservatoire National des Arts et Métiers, Paris, France.

Haradji, Y., \& Faveaux, L. (2006). Évolution de notre pratique de conception (1985-2005): modéliser pour mieux coopérer à partir des critères d'utilité, d'utilisabilité.... @ctivités, 3(1), 67-97.

Harle, R. K., \& Hopper, A. (2008). The potential for location-aware power management. In Proceedings of the 10th international conference on Ubiquitous computing (pp. 302-311). New York, NY, USA: ACM. doi:10.1145/1409635.1409676

Haué, J.-B. (2003). Conception d'interfaces grand public en terme de situations d'utilisation. Le cas $d u$ «Multi-Accès ». Thèse de contrôle des systèmes, Université Technologique de Compiègne, France. http://jbh.lautre.net/recherche/Download/2003-JBH-THESE-COMPLETE.pdf

Haué, J.-B. (2004). Intégrer les aspects situés de l'activité dans une ingénierie cognitive centrée sur la situation d'utilisation. @ctivités, 1(2), 170-194.

I.F.O.P. (2009). Le regard de Français sur la consommation électrique et les compteurs électriques évolués. http://www.ifop.com/?id=946\&option=com_publication\&type=poll

Kaptelinin, V., \& Nardi, B. A. (2006). Acting with technology: Activity theory and interaction design. Cambridge, MA: MIT Press.

Kirsh, D. (1995). The intelligent use of space. Artificial Intelligence, 73(1-2), 31-68. doi:10.1016/0004-3702(94)00017-U

La Valle-Torres, N. (2011). L'organisation temporelle des activités dans l'espace domestique. Interactions, matérialité, technologies. Thèse de doctorat en linguistique, Université Lyon 2, France. https://tel.archives-ouvertes.fr/tel-00600045/file/La_Valle_Torres_these11.pdf

Lacoste, M. (2001). Quand coordonner c'est communiquer. Communication à l'hôpital et coordination des équipes. In A. Borzeix \& B. Fraenkel (Eds.), Langage et Travail. Communication, cognition, action (pp. 323-342). Paris: CNRS Éditions.

Lorino, P. (2009). Concevoir l'activité collective conjointe : l'enquête dialogique. Étude de cas sur la sécurité dans l'industrie du bâtiment. @ctivités, 6(1), 87-110.

Martini, D., Coletti, S., \& Fréjus, M. (2002). Éclairage domestique : étude ergonomique des usages existants et recommandations pour la conception. Clamart, France: EDF R\&D.

Maturana, H. R., \& Varela, F. (1992). The tree of knowledge. The biological roots of human understanding. Boston: Shambhala.

Meyers, R. J., Williams, E. D., \& Matthews, H. S. (2010). Scoping the potential of monitoring and control technologies to reduce energy use in homes. Energy and Buildings, 42(5), 563-569.

Moles, A. A., \& Rohmer, É. (1973). Psychosociologie de l'Espace (1998th ed.). Paris: L'Harmattan.

Mundutéguy, C., \& Darses, F. (2007). Perception et anticipation du comportement d'autrui en situation simulée de conduite automobile. Le travail humain, 70(1), 1-32. doi:10.3917/th.701.0001

Poizat, G. (2006). Analyse en ergonomie cognitive de l'activité collective en tennis de table. Thèse de doctorat en STAPS, Université de Rouen, France. http://tel.archives-ouvertes.fr/

Poizat, G., Fréjus, M., \& Haradji, Y. (2009). Analysis of activity in domestic settings for the design 
ubiquitous technologies. In European Conference on Cognitive Ergonomics (pp. 141-142).

Helsinki, Finland: VTT Technical Research Centre of Finland.

http://dl.acm.org/citation.cfm?id=1690508.1690527

Poizat, G., Fréjus, M., \& Haradji, Y. (2012). Ergonomics at home: Contribution to the design of a smart home lighting service AHFE - Advances in the Human Side of Service Engineering (pp. 8493). San Francisco: CRC Press. http://www.crenetbase.com/doi/abs/10.1201/b12315-12

Ramduny-Ellis, D., Dix, A., Gill, S., \& Hare, J. (2009). Physicality and interaction. Interacting with Computers, 21(1-2), 64-65. doi:10.1016/j.intcom.2008.10.003

Rodden, T. A., Fischer, J. E., Pantidi, N., Bachour, K., \& Moran, S. (2013). At home with agents: exploring attitudes towards future smart energy infrastructures. In Proceedings of the SIGCHI Conference on Human Factors in Computing Systems (pp. 1173-1182). New York, NY, USA: ACM. doi:10.1145/2470654.2466152

Sartre, J.-P. (1943). L'être et le néant. Paris: Gallimard.

Salembier, P., Dugdale, J., Fréjus, M., \& Haradji, Y. (2009). A descriptive model of contextual activities for the design of domestic situations. In European Conference on Cognitive Ergonomics (pp. 131-137). Helsinki, Finland: VTT Technical Research Centre of Finland. http://dl.acm.org/citation.cfm?id=1690508.1690526

Salembier, P., \& Zouinar, M. (2006). Pas de coopération sans partage! Le partage d'information comme régulateur de la cognition individuelle et collective. In F. Jeffroy, J. Theureau, \& Y. Haradji (Eds.), Relation entre activité individuelle et activité collective. Confrontation de différentes démarches d'études (pp. 211-240). Toulouse: Octarès.

Sève, C., Theureau, J., Saury, J., \& Haradji, Y. (2012). Drôles d'endroits pour une rencontre : STAPS, Ergonomie \& Cours d'action. In M. Quidu (Ed.), Les sciences du sport en mouvementinnovations et traditions théoriques en STAPS (pp. 39-64). Paris: L'Harmattan. http://www.coursdaction.fr

Suchman, L. (2007). Human-machine reconfigurations. Plans and situated actions, 2nd edition. New York, N.Y: Cambridge University Press.

Theureau, J. (2004a). Le cours d'action : méthode élémentaire. Toulouse: Octarès.

Theureau, J. (2004b). L'hypothèse de la cognition (ou action) située et la tradition d'analyse du travail de l'ergonomie de langue française. @ctivités, 1(2), 11-25.

http://www.activites.org/v1n2/theureau.pdf

Theureau, J. (2006). Le cours d'action : méthode développée. Toulouse: Octarès.

Theureau, J. (2011). Appropriation, incorporation, in-culturation. Journée Ergo-IdF, Appropriation et Ergonomie. Paris: C.N.A.M. http://www.coursdaction.fr/index.htm

Theureau, J., \& Jeffroy, F. (1994). Ergonomie des situations informatisées : la conception centrée sur le cours d'action. Toulouse: Octarès.

Vermersch, P. (2012). Explicitation et phénoménologie. Paris: PUF.

Zouinar, M., \& Fréjus, M. (2010). Ergonomie des situations domestiques : Nouveaux défis, nouvelles opportunités. In G. Valléry, M.-C. Le Port, \& M. Zouinar (Eds.), Ergonomie, conception de produits et services médiatisés. Paris: PUF.

\section{RÉSUMÉ}

Analyse de contextes d'activité domestique pour la conception de systèmes diffus énergétiquement efficients. À partir d'une approche située de l'activité en ergonomie cognitive, cet article traite de l'ergonomie de futures situations d'informatique diffuse efficientes énergétiquement, à partir d'une analyse des contextes d'activité domestique. La thèse soutenue, issue d'une recherche doctorale, est qu'une telle conception passe a minima par la connaissance empirique de trois types de contextes d'activité : la signification de l'action pour l'acteur, les articulations collectives de ces significations et les agencements physiques de l'activité. Après 
avoir brièvement évoqué les visées de conception, le cadre théorique et méthodologique développé, nous la défendons en deux temps. D'une part, nous montrons en quoi nos résultats empiriques sur ces trois dimensions alimentent la conception en termes de principes de conception, de type d'interaction à privilégier ou de types de contextes utiles au système pour mettre en œuvre ses fonctionnalités, atteindre ses objectifs et finalité. D'autre part, nous montrons que nos avancées méthodologiques et résultats à propos des contextes d'activité permettent de formaliser nos choix de conception de façon explicite. La discussion ouvre des perspectives pour des approches en première personne de l'activité humaine et la caractérisation des espaces physiques.

MOTS-CLEFS

Informatique diffuse, gestion de l'énergie, contexte, activité domestique, méthodologie

\section{RÉFÉRENCEMENT}

Guibourdenche, J., Vacherand-Revel, J., Fréjus, M., \& Haradji, Y. (2015). Analyse de contextes d' activité domestique pour la conception de systèmes diffus énergétiquement efficients. Activités, 12(1), 46-69. http://www.activites.org/v12n1/v12n1.pdf

Article soumis le 22/04/2014, accepté le 13/02/2015 